\title{
Interleukin-17 enhances the removal of respiratory syncytial virus in mice by promoting neutrophil migration and reducing interferon-gamma expression
}

G. Zhang ${ }^{1}$, K.F. Zhou ${ }^{2}$ and Z.H. Lu ${ }^{1}$

1'Department of Critical Care Medicine, Sir Run Run Shaw Hospital, Medical Department of Zhejiang University, Hangzhou, Zhejiang, China

${ }^{2}$ Institute of Viral Disease, Zhejiang Academy of Medical Sciences, Hangzhou, China

Corresponding author: G. Zhang

E-mail: zhangge1512@126.com / zhangge197412@sina.com

Genet. Mol. Res. 15 (1): gmr.15017002

Received August 16, 2015

Accepted October 19, 2015

Published March 24, 2016

DOI http://dx.doi.org/10.4238/gmr.15017002

ABSTRACT. The aim of this study was to observe the effect of interleukin (IL)-17 on early immune response and inflammation in the lungs of respiratory syncytial virus (RSV)-infected mice. Specific pathogen-free BALB/c mice were randomly assigned to control, RSV-infected, RSVinfected with phosphate-buffered saline, and RSV-infected + IL-17 intervention groups. The RSV infection model was set up by nasal mucosa immunization. The intervention group was provided with restructured IL-17 (intranasal). The viral load and cytokine concentrations in the lung tissues and broncho-alveolar lavage fluid (BALF) were determined by real-timepolymerase chain reaction and enzyme-linked immunosorbent assay. RSV caused acute lung inflammation in mice with a significantly higher number of neutrophils and cytokines such as interferon-gamma (IFN- $\gamma$ ), IL-1 $\beta$, IL-6, and G-CSF in the BALF than that in the control group. IL-17 intervention led to a significant increase in the number of neutrophilic granulocytes in the BALF. Alternately, IL-17 intervention led to a significant decrease in 
the IFN- $\gamma$ concentration and a significant increase in the IL-1 $\beta$, IL-6 and G-CSF levels in the BALF. IL-17 induced a reduction in the viral load and an increase in the survival rate of mice on the third day of infection. IL17 mucosal immunity enhances the removal of RSV and strengthens the immune defense by promoting neutrophil migration and reducing the IFN- $\gamma$ levels in mouse lungs.

Key words: Respiratory syncytial virus; Interleukin-17; Interferon-gamma; Neutrophil

\section{INTRODUCTION}

Respiratory syncytial virus (RSV) is one of the most common viruses responsible for respiratory infections in humans. This virus forms a special cell fusion lesion in tissue cultures. RSV infection often causes serious capillary bronchitis and pneumonia (Durbin and Durbin, 2004; Collins and Graham, 2008; Dawson-Caswell and Muncie Jr., 2011). Recent studies have shown that interleukin (IL)-17 plays an important role in viral infection-mediated immune injury. Following infection and in the presence of IL-17, nonimmune cells such as fibroblasts produce a large number of inflammatory factors and chemotactic factors that induce inflammatory cells, such as neutrophils, to infiltrate and damage tissues and organs. Excessive inflammation in the body leads to a pathological increase in infection (Miyamoto et al., 2003; Weaver et al., 2007). The body cannot effectively remove the virus in case of a lack of inflammatory response. Therefore, IL-17 may play a dual role. We constructed a mouse RSV infection model, and investigated the potential role of IL-17 in acute lung injury resulting from RSV infection. We also conducted a preliminary analysis of the mechanism of IL-17 function, and attempted to determine if IL-17 plays a defensive or pathogenic role in host immune defense.

\section{MATERIAL AND METHODS}

\section{Animals and viral strains}

Clean, graded male ICR mice (6 to 8 weeks old) weighing 20 to $25 \mathrm{~g}$ were purchased from the Laboratory Animal Center of the Zhejiang Medical Sciences Academy. All studies were performed in accordance with the American Association for the Accreditation of Laboratory Animal Care guidelines (for humane treatment of animals), and adhered to the national and international standards.

HEP-2 cells were infected with respiratory syncytial virus long strains obtained from Guangzhou Botte Biological Technology Co. Ltd. (Guangzhou, China). The cells were scraped off when fusion lesions (typical of disease development) were observed in $>90 \%$ of the culture; the supernatant was collected by centrifugation of the cells. The cell concentration was adjusted to 1 $\times 10^{7}$ plaque forming units (PFU)/mL and incubated until the formation of plaque viral drops. This was then incubated at $-80^{\circ} \mathrm{C}$ until further use.

\section{Grouping and viral infection in mice}

Eighty mice were randomly divided into four groups ( $N=20$ each). After anesthesia, the mice were infected with $100 \mu \mathrm{L}\left(1 \times 10^{6} \mathrm{PFU}\right) \mathrm{RSV}$ solution. Mice in the control group were infected 
with $100 \mu \mathrm{L}$ HEP-2 cell culture supernatant containing unvaccinated RSV. Mice in the PBS control group were intranasally treated with $50 \mu \mathrm{L}$ 1X PBS $12 \mathrm{~h}$ before the infection. Mice in the IL-17 intervention group also underwent an intranasal treatment of $50 \mu \mathrm{L}$ PBS supplemented with 5 $\mu \mathrm{g}$ recombinant IL-17. The weight, surface wrinkling, ataxia ("+" indicated yes, "-" indicates the absence of ataxia), and pulmonary congestion status ("-" represents congestion area of $0 \%$, "+" $30-70 \%$, and "++" indicates a congestion area $>70 \%$ ) of mice were recorded the corresponding time points after infection.

\section{Detection of viral load in the lung tissue}

The viral load was detected in the RSV-infected lung tissue by real-time polymerase chain reaction (PCR) at specific time points $(1,3,5$, and 7 days). Total RNA was extracted from freshly obtained lung tissues using the TRIzol reagent (Invitrogen, Carlsbad, CA, USA) according to the manufacturer protocols, and cDNA was synthesized by reverse transcription. The synthesized cDNA was used as the template for real-time PCR amplification using the SYBR Ex Script RT-PCR Kit (TaKaRa Bio Inc., Tokyo, Japan). The RSV nucleoprotein gene was analyzed using mice $\beta$-actin mRNA as the internal reference. The viral load after infection at specific time points was determined, taking the viral mRNA/ $\beta$-actin mRNA ratio of the first day after infection as 1.

\section{Hematoxylin-eosin (H\&E) staining of lung tissues}

RSV-infected lung tissues were obtained from mice 3 days after infection; the tissues were embedded in paraffin and sliced using a standard procedure, and stained with H\&E. The sections were then observed under a microscope.

\section{Separation and counting of alveolar lavage fluid cells}

The alveolar space in mice was lavaged with $1 \mathrm{~mL}$ sterile PBS solution at specific time points; the cytokine content in the supernatant of alveolar lavage fluid (obtained by centrifugation) was detected; the supernatant was cryopreserved for future experimentation. The cells collected and resuspended were counted. One hundred microliters of the suspension was placed on a slide, applied evenly, and subjected to H\&E staining. The cell content was classified, and the number of granulocytes, especially neutrophils, was determined by light microscopy.

\section{Enzyme-linked immunosorbent assay (ELISA)}

The concentrations of cytokines in the alveolar lavage fluid obtained from the different groups of mice were determined using standard ELISA kits (Invitrogen).

\section{Statistical methods}

All data were analyzed on the SPSS (v.10.1; IBM, Armonk, NY, USA) statistical platform. All data are reported as means \pm standard deviation (SD). The groups were compared by a standard t-test; $\mathrm{P}<0.05$ was considered statistically significant. 


\section{RESULTS}

\section{RSV infection causes acute lung injury in mice}

Changes in the weights of mice infected with RSV (determined by pathological observation) are shown in Figure $1 \mathrm{~A}$ and Table 1 . The body weight of mice infected with RSV decreased significantly from the time of infection to the third day post-infection; this decrease was especially significant on the first day after inoculation, and was accompanied by arching of the back, wrinkling of fur, decrease in activity and fatigue, and significant bleeding in the lung.

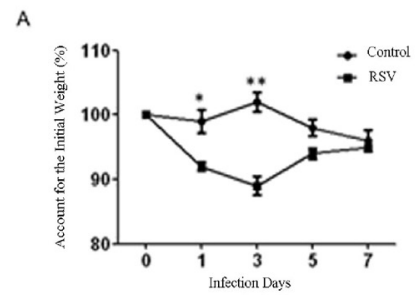

B

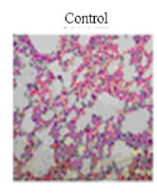

C

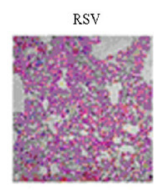

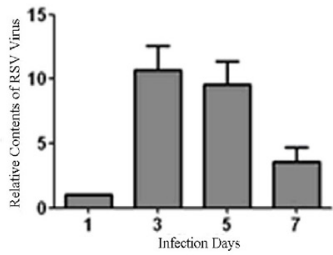

Figure 1. Detection of weight, lung tissue pathology and viral load in mice after respiratory syncytial virus (RSV) infection. A. Weight data by scales of mice. B. H\&E stained in the lung tissue. C. Viral load of RSV-infected.

\begin{tabular}{|c|c|}
\hline & Sequence \\
\hline \multirow[t]{2}{*}{ Nucleoprotein gene } & Upper 5'-GGAACAAGTTGTTGAGGTTTATGAATATGC-3' \\
\hline & Low 5'-CTTCTGCTGTCAAGTCTAGTACACTGTAGT-3' \\
\hline \multirow[t]{2}{*}{$\beta$-actin } & Upper 5'-TGACGTTGACATCCGTAAAGACC-3' \\
\hline & Low 5'-CTCAGGAGGAGCAATGATCTTGA-3' \\
\hline
\end{tabular}

Observations of the H\&E-stained lung tissue revealed an obvious thickening of the alveolar walls; in addition, the alveolar space was significantly infiltrated by lymphocytes, macrophages, and neutrophils (Figure 1B). The RSV-infected viral load was detected within 1 week; we discovered that the viral load increased gradually during the first 3 days and decreased gradually over the last 4 days (Figure 1C). These results revealed that acute lung injury was observed in mice during the early stages of RSV infection.

\section{RSV infection-induced neutrophil accumulation in mice and effect of IL-17 intervention}

The IL-17 level in the broncho-alveolar lavage fluid was detected by ELISA; IL-17 concentration in the alveolar lavage fluid was found to increase significantly in 3-day RSV-infected 
mice than in the control group $(P<0.01)$. Subsequently, there was a gradual decrease in IL-17 level (Figure 2). The IL-17 concentration in the alveolar lavage fluid of mice subjected to advance IL-17 intervention increased significantly on day 1, compared to those in mice of the RSV-infected and non-intervention groups $(P<0.01$; Figure $2 A)$.

The results of cell counting indicated that RSV infection resulted in a significant increase in the neutrophil content in the alveolar lavage fluid of mice, compared to that seen in the control group. The number of neutrophils in the alveolar lavage fluid of RSV-infected mice after IL-17 intervention increased significantly compared to that in untreated mice. Therefore, IL-17 intervention appeared to play a role in the stimulation of neutrophils during the early stages of infection (Figure 2B).

Neutrophil migration-related cytokines, such as IL-1 $\beta$, IL-6, and G-CSF, were observed on the first day of infection by ELISA; additionally, IL-17 was shown to induce the cytokines IL$1 \beta$, IL-6, and G-CSF, which promote the migration of neutrophils (Figure 2C). Contrastingly, the infection group showed significantly different results $(P<0.05)$, suggesting that IL-17 might assist in neutrophil migration through the expression of cytokines that specifically promote this migration.
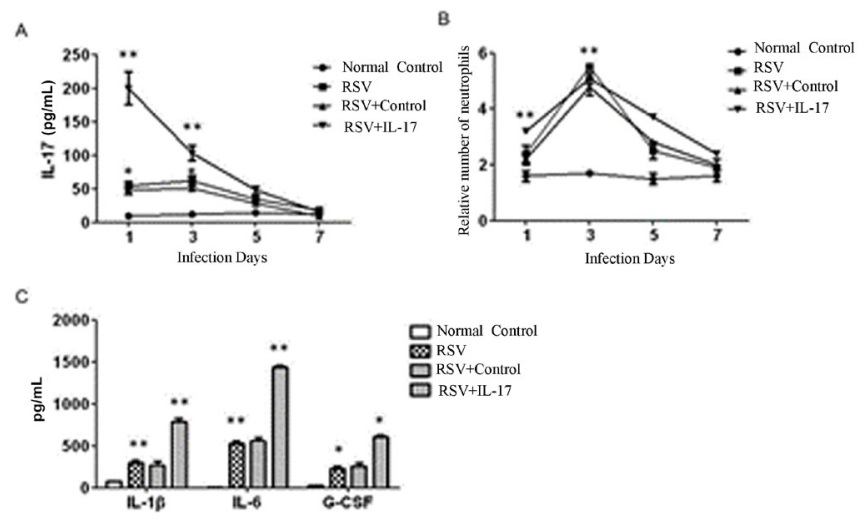

Figure 2. Detection of the neutrophils and the related cytokines in alveolar lavage fluid in respiratory syncytial virus (RSV)-infected mice. A. Level of IL-17 in the broncho-alveolar lavage fluid by ELISA. B. Number of neutrophils in alveolar lavage fluid in mice. C. ELISA was used to detect the neutrophil migration-related cytokines.

\section{Increase in IFN- $\gamma$ concentration in RSV-infected mice and the effect of IL-17 intervention}

We observed a significant increase in the IFN- $\gamma$ concentration in the alveolar lavage fluid of RSV-infected mice during the first and third days of infection, compared to the normal control group $(\mathrm{P}<0.01$; Figure 3). On the other hand, the IFN- $\gamma$ concentration decreased significantly with IL-17 intervention compared to that in the untreated infection group. This suggested that IL-17 exerts a protective effect on acute lung injury in mice by decreasing the IFN- $\gamma$ concentration.

\section{Effect of recombinant IL-17 protein intervention on mouse survival and viral load}

We observed a significant decrease in the viral load in the alveolar lavage fluid of RSVinfected mice after recombinant IL-17 intervention on day 3, compared to that in RSV-infected and non-intervention-infected mice $(P<0.05)$. However, we observed no significant differences in the viral load between RSV-infected and non-intervention-infected mice (Figure 4A). Meanwhile, IL-17 
intervention for a week helped restore the weight of the treated mice (to some extent), as a result of reduced RSV infection (Figure 4B). The mouse survival curve revealed a significant increase in the survival rate of mice treated with IL-17 on day 3, compared to that of RSV-infected and nonintervention-infected mice $(P<0.05$; Figure 4C).

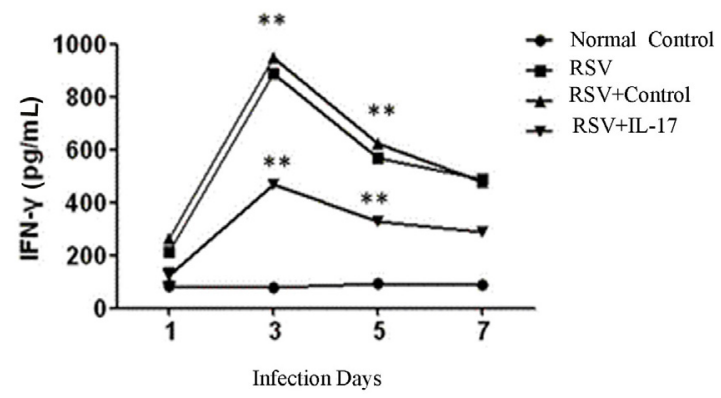

Figure 3. Expression of IFN- $\gamma$ concentration in alveolar lavage fluid in respiratory syncytial virus (RSV)-infected mice, and the effect of IL-17 intervention.
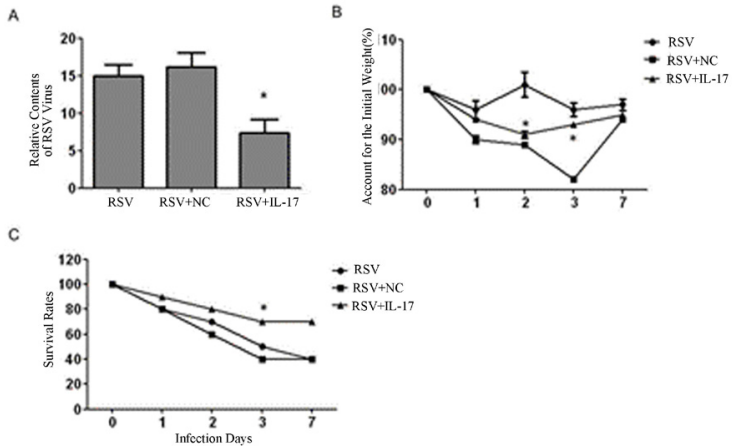

Figure 4. Effect of IL-17 protein intervention on viral load, mouse weight and survival rate. A. Viral load of respiratory syncytial virus (RSV)-infected. B. Weight data by scales of mice. C. Survival rate of the mice. NC = normal control.

\section{DISCUSSION}

Bacterial or viral infection leads to the production of a large number of inflammatory cytokines in the body, that is, moderate inflammation is a natural and effective defense mechanism adopted by the body. On the other hand, excessive inflammation may cause tissue or organ damage. The avian flu virus, SARS virus, and RSV often causes lung infections, and infection of the central nervous system (Ruckwardt et al., 2009; Wissinger et al., 2009). The mechanism by which viral infection initiates the production of a large number of inflammatory cytokines has not been elucidated so far. IL-17 is mainly found in Th17 cells, and was observed in $\gamma \delta \mathrm{T}$ cells for the first time in 1993 (Harrington et al., 2005). Some studies have reported that IL-17 plays an important role in the immune process in host lungs, by recruitment of neutrophils to the local infection site and production of cytokines inducing neutrophil migration by stimulating the cells. This resulted in the effective removal of pathogens from the host (Laan et al., 2002; Dragon et al., 2008; Prause et al., 2009). 
Ryzhakov et al. (2011) reported that the body produces a large number of IL-17 after infection by a virus, and that IL-17 synergistically increases the specificity of inflammation via antiviral signaling pathways. These researchers infected human primary fibroblast HPF cells with RSV, and subsequently observed a significant increase in the mRNA level of various cell inflammatory factors, such as IL-6, IL-8, CXCL1, and CCL8. However, such an effect cannot be achieved by an IL-17 stimulus alone.

The addition of IL-17 to RSV-infected HPF improves the levels of IL- 6 and IL-8 secretion. In this study, an RSV infection model was constructed; additionally, RSV was found to induce acute lung injury, accompanied by an increase in IL-17 concentration in the alveolar lavage fluid, as well as neutrophil accumulation. This suggested that IL-17 participated in the mechanism of RSV infection.

Further study indicated that IL-17 promotes the secretion of cytokines inducing neutrophil migration, such as IL-1 $\beta$, IL-6, and G-CSF, and further recruits neutrophils to the inflammation site in the lung (Huang et al., 2005; Tregoning et al., 2008; Li et al., 2012). This facilitates the removal of virus and an improvement in the survival rate. In conclusion, IL-17 plays a protective role in the process of host immune defense.

Some studies have also shown that acute lung injury due to RSV infection is related to the excessive production of IFN- $\gamma$ by lung NK cells. IL-17 intervention was shown to effectively stem the increase in IFN- $\gamma$ (caused by the RSV infection) concentration in our model. We propose that IL-17 may inhibit the excessive activation of NK cells (or other similar cells) by inhibiting the expression of IFN- $\gamma$, consequently maintaining the inflammatory response of the body and cellular immune response in a relatively balanced and stable state.

Based on these results, we concluded that IL-17 participates in the early immune defense against RSV in infected lungs, by promoting the secretion of cytokines inducing neutrophil migration (IL$1 \beta$, IL-6, and G-CSF) and recruiting neutrophils to the area of inflammation in the lung, or by inhibiting excessive activation of the immune response by reducing the IFN- $\gamma$ content, thereby facilitating virus removal and increasing the survival rate. IL-17 plays a key role in the host immune defense. Therefore, future studies must focus on elucidating the mechanism with which the body regulates the immune response and inflammation response processes. The results of this study also suggested that IL-17 and IL-17R could be used as potential targets for the regulation of host immune defense.

\section{Conflicts of interest}

The authors declare no conflict of interest.

\section{REFERENCES}

Collins PL and Graham BS (2008). Viral and host factors in human respiratory syncytial virus pathogenesis. J. Virol. 82: 20402055. http://dx.doi.org/10.1128/JVI.01625-07

Dawson-Caswell M and Muncie HL Jr (2011). Respiratory syncytial virus infection in children. Am. Fam. Physician 83: 141-146.

Dragon S, Saffar AS, Shan L and Gounni AS (2008). IL-17 attenuates the anti-apoptotic effects of GM-CSF in human neutrophils. Mol. Immunol. 45: 160-168. http://dx.doi.org/10.1016/j.molimm.2007.04.027

Durbin JE and Durbin RK (2004). Respiratory syncytial virus-induced immunoprotection and immunopathology. Viral Immunol. 17: 370-380. http://dx.doi.org/10.1089/vim.2004.17.370

Harrington LE, Hatton RD, Mangan PR, Turner H, et al. (2005). Interleukin -17-producing CD4+ effector T cells develop via a lineage distinct from the T helper type 1 and 2 lineages. Nat. Immunol. 6: 1123-1132. http://dx.doi.org/10.1038/ni1254

Huang KJ, Su IJ, Theron M, Wu YC, et al. (2005). An interferon-gamma-related cytokine storm in SARS patients. J. Med. Virol. 75: 185-194. http://dx.doi.org/10.1002/jmv.20255 
Laan M, Palmberg L, Larsson K and Linden A (2002). Free soluble interleukin-17 protein during severe inflammation in human airways. Eur. Respir. J. 19: 534-537. http://dx.doi.org/10.1183/09031936.02.00280902

Li F, Zhu H, Sun R, Wei H, et al. (2012). Natural killer cells are involved in acute lung immune injury caused by respiratory syncytial virus infection. J. Virol. 86: 2251-2258. http://dx.doi.org/10.1128/JVI.06209-11

Miyamoto M, Prause O, Laan M, Sjöstrand M, et al. (2003). Endogenous IL-17 mediates endotoxin induced airway neutrophilia in mice in vivo. J. Immunol. 170: 4665-4672. http://dx.doi.org/10.4049/jimmunol.170.9.4665

Prause O, Bossios A, Silverpil E, Ivanov S, et al. (2009). IL-17-producing T lymphocytes in lung tissue and in the bronchoalveolar space after exposure to endotoxin from Escherichia coli in vivo - effects of anti-inflammatory pharmacotherapy. Pulm. Pharmacol. Ther. 22: 199-207. http://dx.doi.org/10.1016/j.pupt.2008.12.005

Ruckwardt TJ, Bonaparte KL, Nason MC and Graham BS (2009). Regulatory T cells promote early influx of CD8 T cells in the lungs of respiratory syncytial virus-infected mice and diminish immunodominance disparities. J. Virol. 83: 3019-3028. http://dx.doi.org/10.1128/JVI.00036-09

Ryzhakov G, Blazek K, Lai CC and Udalova IA (2011). IL-17 receptor adaptor protein Act1/CIKS Act1/CIKS plays an evolutionarily conserved role in antiviral signaling. J. Immunol. 187: 5357-5362. http://dx.doi.org/10.4049/jimmunol.1100917

Tregoning JS, Yamaguchi Y, Harker J, Wang B, et al. (2008). The role of T cells in the enhancement of respiratory syncytial virus infection severity during adult reinfection of neonatally sensitized mice. J. Virol. 82: 4115-4124. http://dx.doi. org/10.1128/JVI.02313-07

Weaver CT, Hatton RD, Mangan PR and Harrington LE (2007). IL-17 family cytokines and the expanding diversity of effector T cell lineages. Annu. Rev. Immunol. 25: 821-852. http://dx.doi.org/10.1146/annurev.immunol.25.022106.141557

Wissinger E, Goulding J and Hussell T (2009). Immune homeostasis in the respiratory tract and its impact on heterologous infection. Semin. Immunol. 21: 147-155. http://dx.doi.org/10.1016/j.smim.2009.01.005 